\title{
Nurse Assisted Distraction Strategies (NADS) on Postoperative Pain Experience among Children Undergoing Surgery: A Study Protocol
}

\author{
Edlin Glane Mathias', Mamatha Shivananda Pai ${ }^{2}$, Vijay Kumar ${ }^{3}$, \\ Dinesh Narayanakurup ${ }^{4}$, Malavika $^{5}$, Vasudeva Guddattu ${ }^{6}$ \\ ${ }^{I}$ MPhil (N), PhD Scholar, Manipal College of Nursing, Manipal Academy of Higher Education (MAHE), \\ Manipal, Udupi District, Karnataka, India, ${ }^{2}$ PhD, Professor, Manipal College of Nursing, Manipal Academy \\ of Higher Education (MAHE), Manipal, Udupi District, Karnataka, India, ${ }^{3}$ Professor \& Head, Department \\ of Pediatric Surgery, Kasturba Medical College, Manipal Academy of Higher Education (MAHE), Manipal, \\ Udupi District, Karnataka, India, ${ }^{4}$ Associate Professor, Department of Clinical Psychology, Manipal College of \\ Health Professions, Manipal Academy of Higher Education (MAHE), Manipal, Udupi District, Karnataka, India, \\ ${ }^{5}$ Associate Professor, Department of Anaesthesiology, Kasturba Medical College, Manipal Academy of Higher \\ Education (MAHE), Manipal, Udupi District, Karnataka, India, ${ }^{6}$ Associate Professor, Department of Data Science, \\ Prasanna School of Public Health, Manipal Academy of Higher Education (MAHE), Manipal, Udupi District,
} Karnataka, India

\begin{abstract}
Purpose: To evaluate the efficacy of nurse assisted distraction strategies on postoperative pain, parent pain catastrophizing, mother and child anxiety and physiological parameters.

Method: The present study included a total of 160 mother-child dyads (2-7 years) inpatient to paediatric surgical unit. The study participants are randomized into intervention and control group. The intervention group will receive age appropriate NADS along with the standard care for three postoperative days and the control group will receive only standard care. The participants will be followed from day of admission to postoperative day 3 .

Results: NADS will be delivered for three postoperative days to the children in the postoperative unit. Children received intervention had shown improvement in the postoperative pain and anxiety in these three postoperative days.

Conclusion: The findings of the study will empower nurses and health care professionals to practice distraction strategies in the postoperative unit which will decrease postoperative pain, anxiety and reduces the dosage of analgesics among children.
\end{abstract}

Keywords: Postoperative pain; Anxiety; Children; Nursing, Distraction; RCT; Protocol.

\section{Introduction}

A hospitalized child experience pain more

\section{Corresponding Author:}

\section{Dr. Mamatha Shivananda Pai}

$\mathrm{PhD}$, Professor, Manipal College of Nursing, Manipal

Academy of Higher Education (MAHE), Manipal,

Udupi District, Karnataka, India

e-mail: mamatha.spai@manipal.edu frequently and it is a leading concern of parents and caregivers. ${ }^{1}$ Postoperative pain involves multiple physiological mechanisms precipitated by surgical trauma. ${ }^{2}$ It is associated with autonomic, endocrine, metabolic, physiological and behavioural responses. ${ }^{3}$ Evidence suggests that inadequate relief of postoperative pain results in harmful physiological and psychological consequences that eventually lead to significant morbidity, which may delay recovery and return to daily living and mortality. ${ }^{4}$ It is found that $64 \%$ of the 
post-operative children experience moderate to severe pain and among them 29\% experience unbearable pain after surgery and atleast 24 hours after surgery. ${ }^{5}$ However, pharmacological method are usually practiced in all health care setting to reduce pain which is administered through different routes. ${ }^{6}$ Meanwhile, non-pharmacological techniques are commonly considered additional strategies which are used alone or in combination with pharmacological treatment. ${ }^{7}$ Non-pharmacological interventions provide relaxation and balance the physiological indicators such as blood pressure, pulse rate and respiration and also prevent the post-operative complications such as pain, sleep disorder and anxiety. ${ }^{8}$ To ensure proper management of pain in the post-operative period the pain must be assessed by using the age appropriate tools. ${ }^{9}$ The pain expression of the children is the most appropriate indicator in the assessment of pain and the children those who are not able to communicate pain must be measured by using behavioural pain scales. ${ }^{10}$ Nurses play an effective role in identifying and controlling post-operative pain and teaching the non-pharmacological interventions to the parents and caregivers ${ }^{11}$. Non-pharmacological intervention is often used to guide attention away from painful stimuli. ${ }^{12}$ It is most effective when adapted to the patient's developmental and cognitive level ${ }^{13}$. Most of the studies have reported that non-pharmacological interventions are effective among children when used along with pharmacological treatment. ${ }^{14-20}$ By reviewing the literature, the researchers identified that there are non-pharmacological interventions for pain but structured interventions need to be developed and used for the children to reduce postoperative pain. However, there are research studies conducted in the area of pain assessment but interventions to reduce postoperative pain among younger children is a new concept.

\section{Study objectives:}

\section{The objectives of the study are to:}

- assess the pain, anxiety and physiological parameters of the child inpatient to surgery.

- assess the anxiety and pain catastrophizing among mother of child inpatient to surgery.

- evaluate the efficacy of NADS on pain, pain catastrophizing, anxiety of child and mother and physiological parameters.

Hypotheses: All research hypotheses will be tested at 0.05 level of significance.

$$
\frac{2[z 1-\alpha / 2+z 1-\rho / 2] \sigma^{2}}{d^{2}}
$$

$\mathrm{H}_{1}$ : There will be significant difference in pain, anxiety, physiological parameters and use of pain medication among child undergoing surgery in the intervention group from that of the control group.

$\mathrm{H}_{2}$ : There will be significant difference in anxiety and pain catastrophizing among mother of child undergoing surgery in the intervention group from that of the control group.

\section{Method}

\section{Study design}

A cluster randomized controlled trial

\section{Inclusion and exclusion criteria}

\section{Inclusion criteria:}

\section{Child:}

- inpatient for a minimum three postoperative days

- in the age group of two to seven years

- $\quad$ undergoing urogenital \& other surgeries

- not had any developmental or behavioural disorders

- able to understand and speak Kannada or English

- accompanied by the mother

Mother:

- able to understand and speak Kannada or English

\section{Exclusion criteria:}

\section{Child:}

- undergoing cardiac, head and neck, orthopaedic surgeries.

- mentally retarded and physically challenged

- $\quad$ posted for emergency surgery

\section{Mother:}

- who speaks other than Kannada or English 


\section{Sample size:}

Sample size is calculated by using the following formula,

The total sample for the study is 160 (80 each for experimental and control group).

\section{Data collection:}

Mother in the intervention or control group: On the day of admission, the purpose of the study will be explained to the mother and subject information sheet will be given. Written informed consent will be obtained from the mother which records mother and child participation in the study. The anxiety of the mother is assessed on day of admission and postoperative day 3 by administering the STAI Scale to the mother. On postoperative day 1 and 3 the thoughts and feelings of the mother when her child is in pain is assessed by administering the PCS-P Scale.

Child in the intervention group: On the day of admission the anxiety of the child is assessed by the researcher using mYPAS Scale. On postoperative day 1the pain and physiological parameters will be assessed after 2 hours of surgery. Next pain and physiological parameters will be assessed every fourth hourly. After 6 hours of surgery the researcher will assess the sedation level of the child using Ramsay Sedation Scale and the child receives an age appropriate distraction intervention for thirty minutes. Each day a new distraction activity is provided for the child. To avoid cross-infection all the toys will be cleaned with the help of Dettol solution and the contaminated toys will be replaced with a new kit. After completion of distraction activity, the child is asked to relax and the pain and physiological parameters will be reassessed after 15 minutes. Onpostoperative day two and three the pain and physiological parameters will be assessed every seventh hourly and the distraction activity is given twice a day.

Child in the control group: All assessments are done same as the intervention group but the child receives no any distraction activity and the child will receive standard care in the postoperative unit.

\section{Data collection tools:}

1. Demographic Proforma: Demographic Proforma was developed by the researcher to collect data on sample characteristics. The tool was divided into sample characteristics and clinical data. Sample characteristics measured: age of mother and child, gender of the child, income, education of parents, previous history of surgery. Clinical data measured type of surgery, type of analgesic, demand for analgesics, pre-medications and duration of surgery.

2. Developmental psychopathology checklist for children: It is a standardized scale developed by Malavika Kapur to measure the behavioural/ developmental disorders among children. For the present study the tool has been modified withthe permission of author. The checklist consists of 56 items which contains dichotomous questions (yes/no) and the areas assessed are developmental history, developmental problems, attention deficit hyperactivity, conduct disorders, learning difficulties, emotional disordersand OCD.Inter-class correlation co-efficient via analysis of variance was $0.965 .^{21}$

3. State trait Anxiety inventory (STAI): It is a standardized tool developed by Charles D Spielberger to measure the state and trait anxiety. STAI form Y1 iscomposed of 20 items to measure state anxiety. State anxiety identifies the anxiety in the person by verbalizing the stress, tension and feeling "at that moment". STAI-Y2 is composed of 20 items concerning trait anxiety which indicates the "general feeling of the person". The higher the score is the greater the anxiety. The reliability obtained in the present study was STAI Form Y1 $=0.93$, STAI Form $Y 2=0.91$.

4. Modified Yale preoperative anxiety scale (mYPAS): It is a standardized tool developed by faculties of Yale University School of Medicine. ${ }^{22-23}$ The scale consists of 22 items divided into five subscales: activity, vocalizations, emotional expressivity, state of apparent arousal and use of parents. Each of the 5 categories has an individual score, ranging from the least anxious, to the most anxious behaviours. The higher the number indicates the more anxious behaviour of the child. Reliability of the tool revealed that inter observer agreement ranged from 0.68 to $0.86 .{ }^{24}$ Researcher has assessed the reliability of the tool by using inter-observer method and agreement was 0.92 which shows the tool is reliable.

5. Ramsay sedation scale (RSS): It is a standardized scale which is used to monitor the level of sedation. The tool contains six items and the scoring of the tool 
and higher the score indicates high level of sedation. Cronbach's alpha of the tool showed internal consistency $\alpha=0.82 .{ }^{25}$ In the present study the researcher has assessed the reliability of the tool by using Cronbach's alpha and the internal consistency was 0.8 which reports the tool is reliable.

6. Evendol pain scale: It is a standardized instrument developed by Fournier-Charriere to measure the postoperative pain among younger children. ${ }^{10}$ The tool is composed of five subscales: vocal or verbal expression, facial expression, movements, postures, interaction with the environment. The scale contains score from 0 to 3 for each item: 0 indicates $=$ sign absent, $1=$ sign weak or transient, $2=$ sign moderate or present about half the time, $3=$ sign strong or present almost all the time. Inter-rater reliability showed kappa $0.7-0.9 .{ }^{26} \mathrm{On}$ postoperative day 1 the researcher will take the pain measurements after 2 hour of surgery and then every fourth hourly. On postoperative day two and three pain is measured every seventh hourly. If the analgesic is administered to oral or rectal route pain is assessed after 45 minutes, 10 minutes if administered through IV route.

7. Pain catastrophizing scale-Parent version (PCS-P): It is a standardized scale developed by Michael JL Sullivan to assess the negative thinking associated with pain. ${ }^{27}$ It includes 13 items which are rated on a 5-point scale ranging from $0=$ "not at all true" to $4=$ "very true". The items are divided into three subscales: rumination-4, magnification-3 and helplessness-6. Items are summed across subscales to derive a total score ranging from 0-52. Higher scores reflect higher levels of catastrophic thinking. In the present study the researcher has assessed the reliability of the tool by using Cronbach's alpha and the internal consistency of the tool is 0.9 which reports the tool is reliable.

8. Physiological parameters: The Heart rate, Respiratory rate and Oxygen saturation is measured by using Philips cardiac monitor (Intellivue MP5). Researcher has assessed the reliability of the monitor by using inter-observer method and agreement was 1 which shows that it is reliable.

Data analysis: EZR software will be used for data analysis. Descriptive statistics will be used for analysing sample characteristics. Repeated measure ANOVA to compare effect of intervention on continuous outcome.

Ethical Clearance: The protocol has been approved by the institutional Ethics Committee (IEC: 35/2019). The study has been registered under Clinical Trial Registry India (CTRI/2019/05/019174).

Table 1: Schematic representation of research methodology

\begin{tabular}{|c|c|c|c|c|c|}
\hline & Group & On admission & Postoperative day 1 & Postoperative day 2 & Postoperative day 3 \\
\hline \multirow{6}{*}{$\begin{array}{l}\text { Cluster } \\
\text { randomization }\end{array}$} & \multirow[t]{3}{*}{$\begin{array}{l}\text { Experimental } \\
\text { group }\end{array}$} & $\begin{array}{c}\text { DPCL } \\
\text { STAI } \\
\text { mYPAS }\end{array}$ & $\begin{array}{c}\text { RSS } \\
\text { Pain } \\
\text { BPP } \\
\text { PCS-P }\end{array}$ & $\begin{array}{l}\text { Pain } \\
\text { BPP }\end{array}$ & $\begin{array}{c}\text { Pain } \\
\text { BPP } \\
\text { PCS-P } \\
\text { mYPAS } \\
\text { STAI }\end{array}$ \\
\hline & & & \multicolumn{3}{|c|}{ Intervention } \\
\hline & & $\mathrm{O}_{1}$ & $\mathrm{O}_{2}$ & $\mathrm{O}_{3}$ & $\mathrm{O}_{4}$ \\
\hline & \multirow[t]{3}{*}{ Control group } & $\begin{array}{c}\text { DPCL } \\
\text { STAI } \\
\text { mYPAS }\end{array}$ & $\begin{array}{c}\text { RSS } \\
\text { Pain } \\
\text { BPP } \\
\text { PCS-P }\end{array}$ & $\begin{array}{l}\text { Pain } \\
\text { BPP }\end{array}$ & $\begin{array}{c}\text { Pain } \\
\text { BPP } \\
\text { PCS-P } \\
\text { mYPAS } \\
\text { STAI }\end{array}$ \\
\hline & & & \multicolumn{3}{|c|}{ Standard Care } \\
\hline & & $\mathrm{O}_{1}$ & $\mathrm{O} 2$ & $\mathrm{O}_{3}$ & $\mathrm{O}_{4}$ \\
\hline
\end{tabular}




\section{Keywords:}

STAI: State Trait Anxiety Inventory

BPP: Bio-physiological parameters

mYPAS: modified Yale Pre-operative Anxiety Scale

DPCL: Developmental Psychopathology checklist for children (DPCL)PCS-P: Pain catastrophizing parent version

\section{Discussion}

Standardized age appropriate distraction strategies have a potential to reduce postoperative pain and anxiety among the child undergoing surgery. The current study presents an opportunity to evaluate such program among the children in the postoperative unit. The strength of the study is using standardized scales for measuring the outcomes. This is the first study where the pain is assessed using Evendol pain scale and the child receives standardized age appropriate distraction strategies intervention for postoperative pain among children. The outcomes of the study will support the training of nurses and health professionals regarding the use of pain scale for assessing postoperative pain and delivering the age appropriate distraction strategies for the child in the postoperative unit.

\section{Conclusion}

NADS will help to reduce postoperative pain and anxiety. The findings of this study will motivate the health professionals to practice distraction strategies in the postoperative unit which benefits the child and family undergoing surgery.

\section{References}

1. Heinrich M, Mechea A, Hoffmann F. Improving postoperative pain management in children by providing regular training and an updated pain therapy concept. Eur J Pain (United Kingdom). 2016;20(4):586-93.

2. Lee JY, Jo YY. Attention to postoperative pain control in children. Korean $\mathrm{J}$ Anesthesiol. 2014;66(3):183-8.

3. Cowen R, Stasiowska MK, Laycock H, Bantel C. Assessing pain objectively: The use of physiological markers. Anaesthesia. 2015;70(7):828-47.

4. Srouji R, Ratnapalan S, Schneeweiss S. Pain in Children: Assessment and Nonpharmacological
Management. Int J Pediatr. 2010;

5. Jimenez N, Jackson DL, Zhou C, Ayala NC, Ebel BE. Postoperative pain management in children, parental english profi ciency, and access to interpretation. Hosp Pediatr. 2014;4(1):23-30.

6. Kahsay H. Assessment and treatment of pain in pediatric patients. Curr Pediatr Res. 2017;21(1):148-57.

7. Eke GK, Briggs DC. Management of Paediatric Pain: Knowledge and Practice of Healthcare Providers at a Tertiary Centre, Southern Nigeria. Asian J Pediatr Res. 2019;2(August 2017):1-8.

8. Subramaniam SD, Doss B, Chanderasekar LD, Madhavan A, Rosary AM. Scope of physiological and behavioural pain assessment techniques in children - A review. Healthc Technol Lett. 2018;5(4):124-9.

9. Brahmbhatt A, Adeloye T, Ercole A, Bishop SM, Smith HL, Wheeler DW. Assessment of postoperative pain in children: Who knows best? Pediatr Rep. 2012;4(1):0-3.

10. Beltramini A, Milojevic K, Pateron D. Pain assessment in newborns, infants, and children. Pediatr Ann. 2017;46(10):e387-95.

11. He HG, Lee TL, Jahja R, Sinnappan R, Vehviläinen-Julkunen K, Pölkki T, et al. The use of nonpharmacological method for children's postoperative pain relief: Singapore nurses' perspectives. J Spec Pediatr Nurs. 2011;16(1):2738.

12. Goettems ML, Zborowski EJ, Costa F dos S, Costa VPP, Torriani DD. Nonpharmacologic Intervention on the Prevention of Pain and Anxiety During Pediatric Dental Care: A Systematic Review. Acad Pediatr. 2017;17(2):110-9.

13. Klausen SH, Rønde G, Tornøe B, Bjerregaard L. Nonpharmacological interventions addressing pain, sleep, and quality of life in children and adolescents with primary headache: A systematic review. J Pain Res. 2019;12:3437-59.

14. Kaheni S, Rezai MS, Bagheri-Nesami M, Goudarzian AH. The effect of distraction technique on the pain of dressing change among 3-6 year-old children. Int J Pediatr. 2016;4(4):1603-10.

15. Gupta HV, Gupta VV, Kaur A, Singla R, Chitkara $\mathrm{N}$, Bajaj K V., et al. Comparison between the analgesic effect of two techniques on the level of pain perception during venipuncture in children up 
to 7 years of age: A quasi-experimental study. J Clin Diagnostic Res. 2014;8(8):1-4.

16. Martin V. Using distraction techniques with children. Nursing (Lond). 2013;43(11):68.

17. Concepción M, Guerrero G. Video-Distraction System to Reduce Anxiety and Pain in Children Subjected to Venipuncture in Pediatric Emergencies. Pediatr Emerg Care Med Open Access [Internet]. 2016;1(1):1-8. Available from: http://pediatricemergency-care.imedpub.com/archive.php

18. Sunitha Suresh BS, De Oliveira GS, Suresh S. The effect of audio therapy to treat postoperative pain in children undergoing major surgery: a randomized controlled trial. Pediatr Surg Int. 2015;31(2):197201.

19. Shaker N, Taha A. Effectiveness of Distraction Therapy on Children's Pain Perceptions During Peripheral Venous Cannulation at Pediatric Teaching Hospital in Erbil City. Erbil J Nurs Midwifery. 2018;1(2):74-83.

20. Sahiner NC, Bal MD. The effects of three different distraction method on pain and anxiety in children. J Child Heal Care. 2016;20(3):277-85.

21. Aswathy KL, Kunju PAM, Mini S. Factors associated with behavioural problems among children receiving anti-epileptic drugs. 2018; $4(1): 18-23$.
22. Kain ZN, Mayes LC, Cicchetti D V., Bagnall AL, Finley JD, Hofstadter MB. The Yale Preoperative Anxiety Scale: How does it compare with a "gold standard"? Anesth Analg. 1997;85(4):783-8.

23. Rema JP, Cavaleiro C, Pina P, Machado HS. Preoperative Anxiety in Preschool ChildrenObservational Study. J Anesth Clin Res. 2016;7(9):1-6.

24. Greeley WJ, Kain ZN, Mayes LC, Cicchetti D V, Bagnall AL, Finley JD, et al. The Yale Preoperative Anxiety Scale: How Does It Compare with a "Gold Standard"? 1997;

25. Rasheed A, Amirah M, Abdallah M, Pj P, Issa M. iMedPub Journals RAMSAY Sedation Scale and Richmond Agitation Sedation Scale (RASS): A Cross Sectional Study. 2018;1-5.

26. Fournier-Charrière E, Tourniaire B, Carbajal $\mathrm{R}$, Cimerman P, Lassauge F, Ricard C, et al. EVENDOL, a new behavioral pain scale for children ages 0 to 7 years in the emergency department: Design and validation. Pain. 2012;153(8):1573-82.

27. Sullivan MJL, Bishop SR, Pivik J. The Pain Catastrophizing Scale: Development and Validation. Psychol Assess. 1995;7(4):524-32. 